\title{
Implementation of underwater precise navigation system for a re- motely operated mine disposal vehicle ${ }^{\dagger}$
}

\author{
Kihun Kim $^{1 *}$, Chong-Moo Lee ${ }^{1}$, Hyun-Taek Choi ${ }^{1}$ and Pan-Mook Lee ${ }^{1}$ \\ ${ }^{1}$ MOERI, KORDI , 171 Jang-dong, Yuseong-gu, Daejeon 303-343, Korea
}

(Manuscript Received February 21, 2010; Revised March 28, 2011; Accepted May 9, 2011)

\begin{abstract}
This paper describes the implementation of a precise underwater navigation solution using a multiple sensor fusion technique based on USBL, GPS, DVL and AHRS measurements for the operation of a remotely operated mine disposal vehicle (MDV). The estimation of accurate $6 \mathrm{DOF}$ positions and attitudes is the key factor in executing dangerous and complicated missions. To implement the precise underwater navigation, two strategies are chosen in this paper. Firstly, the sensor frame alignment to the body frame is conducted to enhance the performance of a standalone dead-reckoning algorithm. Secondly, absolute position data measured by USBL is fused to prevent cumulative integration error. The heading alignment error is identified by comparing the measured absolute positions with the DR algorithm results. The performance of the developed approach is evaluated with the experimental data acquired by MDV in the South-sea trial.
\end{abstract}

Keywords: Dead reckoning (DR), Underwater navigation, Sensor fusion, Ultra short base line(USBL), Doppler velocity $\log (\mathrm{DVL})$

\section{Introduction}

Precise navigation is essential in implementing underwater missions. The performance of a navigation sensor or types of algorithms can be evaluated in terms of three aspects: accuracy, precision and update rate. Accuracy refers to the degree to which the estimated values coincide with the real values. Precision refers to the degree of fineness of resolution that can be achieved. The update rate refers to the rapidity at which the estimation value can be provided. Accuracy is especially important in a wide area survey while precision and the update rate are essential in feedback control for attitude and waypoint tracking, image mosaic and system identification.

Concerning underwater navigation, the submerged body can be described by the 6 DOF mo-

"Corresponding author. Tel.: +82-42-866-3814, Fax.: +82-42-866-3819. E-mail address: shaton@moeri.re.kr.

Copyright $($ KSOE 2011. tion (surge, sway, heave, roll, pitch, and yaw) as well as by altitude. Usually, attitudes, depth, and altitude can be measured quite precisely with a sufficient update rate by commercial sensors such as FOG-AHRS (Fiber optic gyro based attitude heading reference system, $100 \mathrm{~Hz}$ ), a pressure sensor $(20 \mathrm{~Hz})$, and DVL $(5 \mathrm{~Hz})$. To achieve precise full state navigation information, the horizontal positions (north and east in the navigation frame) should be carefully examined. Sensors that measure horizontal motions can be divided into two groups. One group measures the relative motions to the navigation frame fixed on the earth such as the inertial motion unit (acceleration and angular velocity), and the Doppler velocity log (velocity). This group shows good performances in terms of precision and update rate. The other group measures absolute positions such as acoustic positioning systems (USBL, LBL, and SBL). This group has merits in terms of accuracy. Multiple sensor fusion can be a good solution to achieve an accurate and precise 
performance with a sufficient update rate by fusing the physical sensor characteristics of these two group.

Many researches have been conducted to enhance navigation performance in the horizontal plane. Whitcomb et al [1] proposed a Doppler based navigation and investigated that the bottom rock navigation error is mainly due to heading estimation error and that in-situ calibration is needed. Kinsey and Whitcomb [2] enhanced the in-situ calibration technique by comparing the absolute position and standalone bottom rock DR. Jouffroy and Opderbecke [3] designed a diffusion PDE (Partial differential equation) based observer to fuse USBL data and DR results. Cho and Choi [4] designed the SPRHKF (sigma point receding horizon Kalman filter) filter to provide good quality navigation information in a low cost DR/GPS integration solution for seamless land navigation. Cho investigated various Kalman filters such as EKF (extended Kalman filter), RHKF (receding horizon Kalman filter), SPKF (sigma point Kalman filter), and finally SPRHKF to analyze filter performance in various simulation conditions. P. Lee et al [5] proposed an integrated navigation solution to improve the performance of conventional inertial-acoustic navigation systems by adding complementary acoustic range sonar.

In this paper, the authors have adopted an improved fast covariance intersection method for the multiple sensor data fusion that was proposed by Fränken and Hüpper [6]. This fusion scheme is chosen because its merits include being robust, fast, and easy to implement in online.

For outlier rejection of acoustically measured position data smoothing, Vike and Jouffroy [7] proposed a diffusion PDE based observer to reject outliers in post processing. Cleveland [8] proposed a robust locally weighted regression and smoothing scheme.

In this paper, the outlier rejection algorithm has been designed and executed concurrently with a multi-sensor data fusion algorithm.

Fig. 1 depicts the sensor fusion based navigation strategy adopted in this paper. The navigation algorithm can be divided into three subroutines. The first routine is a standalone DR algorithm enhancement. The second routine is the outlier rejection algorithm. The third routine involves the multi- sensor data fusion of measured estimation candidates.

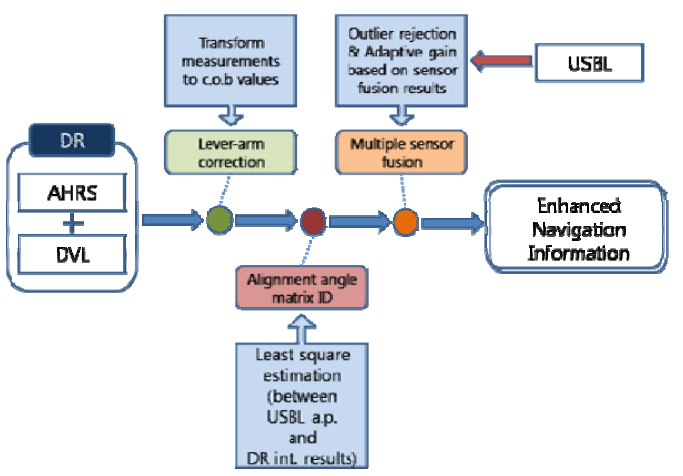

Fig. 1. Sensor fusion based navigation algorithm

\section{DR based underwater navigation}

\subsection{Kinematic formulation}

The body-fixed velocity vector $\mathbf{v}_{\mathbf{o}}^{\mathbf{b}}$ is decomposed in the NED reference frame as: (Fossen [9])

$$
\dot{\mathbf{p}}^{\mathbf{n}}=\mathbf{R}_{\mathbf{b}}^{\mathbf{n}}(\boldsymbol{\Theta}) \mathbf{v}_{\mathbf{o}}^{\mathbf{b}}
$$

where $\dot{\mathbf{p}}^{\mathbf{n}}$ is the velocity vector in the NED frame.

Expanding (1) yields:

$$
\begin{aligned}
\dot{\mathbf{n}}= & u \cos \psi \cos \theta+\mathrm{v}(\cos \psi \sin \theta \sin \phi-\sin \psi \cos \phi) \\
& +\mathrm{w}(\sin \psi \sin \phi+\cos \psi \cos \phi \sin \theta) \\
\dot{\mathrm{e}}= & \mathrm{usin} \psi \cos \theta+\mathrm{v}(\cos \psi \cos \phi+\sin \phi \sin \theta \sin \psi) \\
& +\mathrm{w}(\sin \theta \sin \psi \cos \phi-\cos \psi \sin \phi)
\end{aligned}
$$

Based on the assumption that $\boldsymbol{\phi}$ and $\boldsymbol{\theta}$ are small, Eqs. (2) and (3) can be rewritten as follows:

$$
\begin{gathered}
\dot{\mathrm{n}}=u \cos \psi-v \sin \psi \\
\dot{\mathrm{e}}=u \sin \psi+v \cos \psi
\end{gathered}
$$

where $\mathrm{u}$ and $\mathrm{v}$ are velocities measured at the body fixed point $\mathrm{o}$. And $\dot{\mathrm{n}}$ and $\dot{\mathrm{e}}$ are velocities in the navigation frame.

\subsection{Alignment angle and lever arm identification for compensation}

To implement a precise DR based navigation algorithm, errors especially due to the alignment angle and lever arm should be compensated correctly. 
Fig. 2 depicts the kinematic relation between each sensor frame and the body fixed reference point 0.

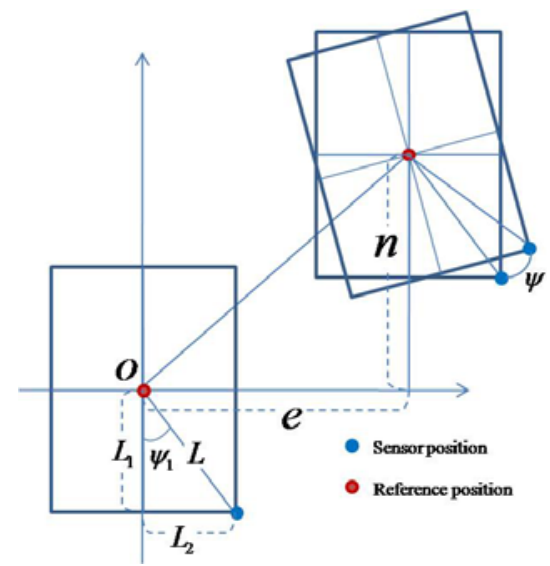

Fig. 2. Kinematic relation between sensor frame and body fixed reference frame

$$
\begin{aligned}
& \mathbf{L}=\sqrt{\mathbf{L}_{1}^{2}+\mathbf{L}_{2}^{2}} \\
& \boldsymbol{\Psi}_{1}=\operatorname{atan} \frac{\mathbf{L}_{2}}{\mathbf{L}_{1}}
\end{aligned}
$$

where $L$ is the lever arm length and $\boldsymbol{\Psi}_{\mathbf{1}}$ is the yaw alignment angle. These are constant values and can be identified by a general least square method.

$$
\begin{aligned}
& \mathrm{e}^{\mathrm{s}}=\mathrm{e}^{\mathrm{b}}+\mathbf{L} \sin \left(\boldsymbol{\psi}+\boldsymbol{\psi}_{1}\right) \\
& n^{s}=n^{b}-L \cos \left(\Psi+\Psi_{1}\right) \\
& \mathrm{e}^{\mathrm{b}}=\mathrm{e}^{\mathrm{s}}-\operatorname{Lsin}\left(\Psi+\Psi_{1}\right) \\
& n^{b}=n^{s}+\operatorname{Lcos}\left(\psi+\Psi_{1}\right) \\
& \dot{\mathrm{e}}^{\mathrm{b}}=\dot{\mathrm{e}}^{\mathrm{s}}-\mathbf{L} \cos \left(\boldsymbol{\Psi}+\boldsymbol{\psi}_{1}\right) \\
& \dot{\mathbf{n}}^{\mathbf{b}}=\dot{\mathbf{n}}^{\mathbf{s}}-\operatorname{Lsin}\left(\Psi+\Psi_{1}\right)
\end{aligned}
$$

Absolute positions measured by the USBL sensor should be transformed using the relation of Eq. (9) and velocities measured by the DVL and IMU sensors should also be transformed using Eqs. (4), (5) and (10) into the respective body fixed reference point values.

\section{Multi-sensor fusion algorithm}

A combined estimate $\hat{\mathrm{x}}$ with error variance matrix $\mathrm{P}$ can be estimated starting with the two observable estimates $\hat{x}_{1}$ and $\hat{x}_{2}$ of the true state $x$ with the corresponding positive definite error va- riance matrices $\mathrm{P}_{1}$ and $\mathrm{P}_{2}$. For uncorrelated estimation errors of MMSE(minimum mean square error) estimates $\hat{\mathrm{x}}_{1}$ and $\hat{\mathrm{x}}_{2}$, the overall MMSE estimate is given by a basic convex combination of the two estimates (Fränken and Hüpper [6]),

$$
\begin{gathered}
\hat{\mathbf{x}}=\mathbf{P P}_{1}^{-1} \hat{\mathbf{x}}_{1}+\mathbf{P P}_{2}^{-1} \hat{\mathbf{x}}_{2} \\
\mathbf{P}^{-1}=\mathbf{P}_{1}^{-1}+\mathbf{P}_{2}^{-1}
\end{gathered}
$$

It is well known that in the case of correlation between the two initial estimation errors, the estimated P may become excessively optimistic and that this can finally cause divergence in sequential filtering.

A conservative estimate can be given without any detailed knowledge about the correlation by applying a covariance intersection as follows:

$$
\begin{gathered}
\widehat{\mathbf{x}}=\omega_{1} P P_{1}^{-1} \widehat{\mathbf{x}}_{1}+\omega_{2} P P_{2}^{-1} \widehat{x}_{2} \\
P^{-1}=\omega_{1} P_{1}^{-1}+\omega_{2} P_{2}^{-1}
\end{gathered}
$$

with nonnegative coefficients $\boldsymbol{\omega}_{1}$ and $\boldsymbol{\omega}_{2}$ obeying

$$
\omega_{1}+\omega_{2}=1
$$

Herein, the coefficients $\omega_{1}$ and $\omega_{2}$ are usually chosen to minimize either the trace or the determinant of $\mathrm{P}$.

Finding the solution of a highly nonlinear optimization problem such as Eq.(12) needs considerable numerical implementation effort. To avoid this, Niehsen has proposed the use of a fast approximate solution instead (Niehsen [10]). When $\operatorname{trace}\left(\mathrm{P}_{1}\right) \ll$ $\operatorname{trace}\left(\mathrm{P}_{2}\right)$, one would expect to obtain $\omega_{1} \approx 1$ to minimize the overall trace.

$$
\omega_{1} \operatorname{trace}\left(P_{1}\right)-\omega_{2} \operatorname{trace}\left(P_{2}\right)=0
$$

which, with (12c), is given by

$$
\omega_{1}=\frac{\operatorname{trace}\left(\mathbf{P}_{2}\right)}{\operatorname{trace}\left(\mathbf{P}_{1}\right)+\operatorname{trace}\left(\mathbf{P}_{2}\right)}
$$

where $\mathbf{0}<\boldsymbol{\omega}_{\mathbf{1}}<\mathbf{1}$.

In Eq. (14), the trace can be replaced by the determinant of the preference instead.

$$
\omega_{1}=\frac{\operatorname{det}\left(\mathbf{P}_{2}\right)}{\operatorname{det}\left(\mathbf{P}_{1}\right)+\operatorname{det}\left(\mathbf{P}_{2}\right)}
$$

In this paper, the authors propose a new 
scheme which has a receding horizon strategy to cope with the changing sensor status as follows:

$$
\begin{aligned}
\hat{\mathbf{x}}= & \omega_{1 \mathrm{k}} \mathbf{P}_{\mathrm{k}} \mathrm{P}_{1 \mathrm{k}}^{-1} \hat{\mathbf{x}}_{1}+\omega_{2 \mathrm{k}} \mathbf{P}_{\mathrm{k}} \mathrm{P}_{2 \mathrm{k}}^{-1} \hat{\mathbf{x}}_{2} \\
& P_{\mathrm{k}}^{-1}=\omega_{1 \mathrm{k}} \mathbf{P}_{1 \mathrm{k}}^{-1}+\omega_{2 \mathrm{k}} P_{2 \mathrm{k}}^{-1}
\end{aligned}
$$

with non-negative coefficients $\boldsymbol{\omega}_{1 \mathrm{k}}$ and $\boldsymbol{\omega}_{2 \mathrm{k}}$ obeying

$$
\omega_{1 \mathrm{k}}+\omega_{2 \mathrm{k}}=1
$$

where subscript $\mathrm{k}$ refers to the value determined with the design data span (computed with $\mathrm{n}$ data). For example, $\mathbf{P}_{\mathbf{k}}$ can be calculated as $\sum_{\mathbf{i}=\mathbf{k}-\mathbf{n}+\mathbf{1}}^{\mathbf{k}} \mathbf{P}(\mathbf{i})$.

In this research, the $\hat{x}_{1}$ can be a local deadreckoning estimate and $\hat{\mathrm{x}}_{2}$ can be an outlier rejected USBL or DGPS measurement for the navigation solution $\hat{\mathrm{x}}$.

\section{Outlier rejection algorithm}

In this study, an intuitive outlier rejection algorithm is proposed. The basic idea originate from the robust local regression method (Cleveland [8]). Cleveland's algorithm can be effectively applied to USBL positioning to remove outliers. However, calculation time is a burden due to the iterative calculating structure.

The proposed scheme for robust weights is given by the simple bi-square function shown below.

$$
\omega_{i}=\left\{\begin{array}{cc}
1 & \left|r_{i}\right|<3 \gamma \\
0 & \left|r_{i}\right| \geq 3 \gamma
\end{array}\right.
$$

$r_{i}$ is the residual of the ith USBL data point produced by the multi-sensor fusion results, and $\gamma$ is the RMS error which can be measured during USBL calibration, for example, $0.3 \%$ of the slant range. The number 3 in front of $\gamma$ is the design factor deciding a watch circle size.

\section{Mine disposal vehicle}

Fig.3 demonstrates a mission scenario of MDV. Its mission is to install ECP(Explosive cutter for Pluto Plus) to the rope of the moored mine and to drop CAP(Charge anti-mine for Plu- to Plus) near the sea bottom mine. To accomplish a complicated and dangerous mission, precise navigation is an essential function which MDV should include. Underwater positions are estimated by the USBL and DVL based navigation system. While surface positions are tracked by DGPS and DVL based navigation solutions. These are overviewed in detail in the next section.

Fig. 4 shows three dimensional computer aided design, where the ECP and CAP can be observed. The ECP is at the upper head part and the CAP is at the under head part of MDV.

Six thrusters generate thrust power to control 5DOF motions except roll motion.

Fig. 5 shows MDV under functional tests. Two types of forward looking sonar systems and a video camera are entrained in the transparent hemi-sphere to detect and identify underwater mine systems against turbid water.

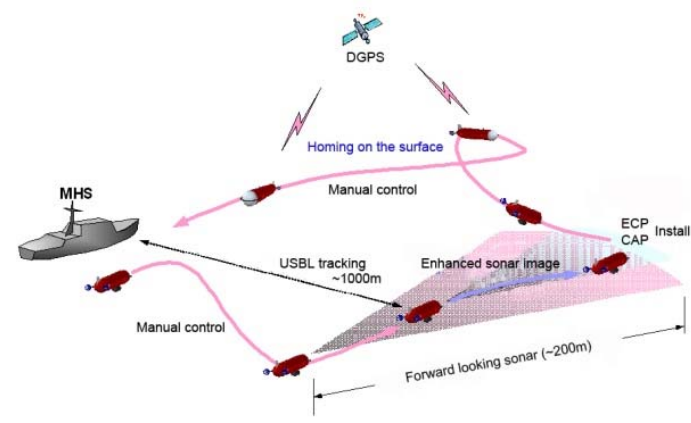

Fig. 3. Mission scenario of MDV

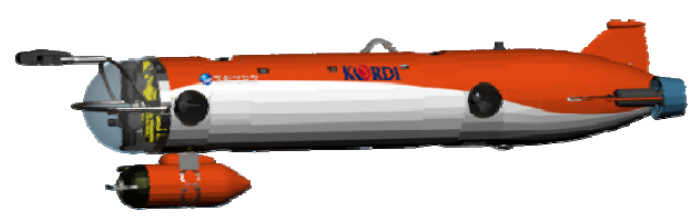

Fig. 4. 3D CAD image of MDV with ECP and CAP 


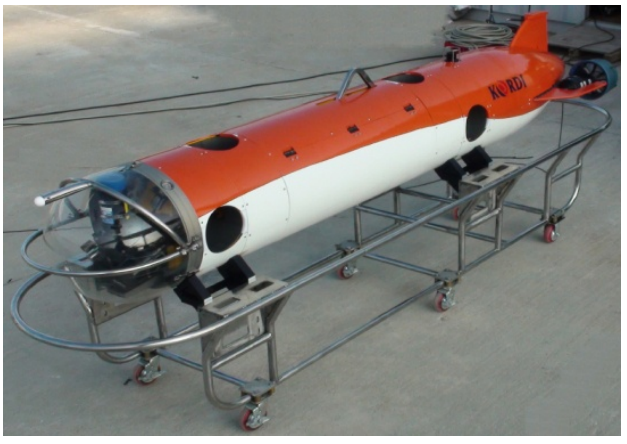

Fig. 5. 3D CAD image of MDV with ECP and CAP

\section{MSF based navigation algorithm results}

\section{and discussions}

\subsection{DGPS, DVL, AHRS fusion algorithm}

Section 6.1 deals with a multi-sensor fusion algorithm for the surface operation state based on DGPS, DVL and AHRS sensors.
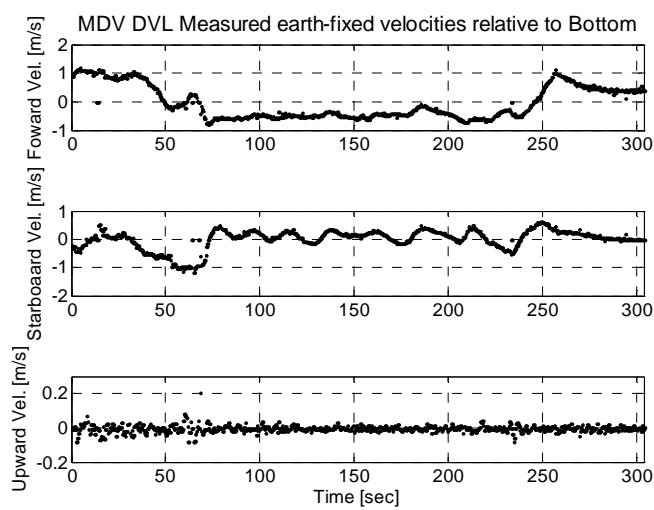

Fig. 6. Measured earth-fixed velocities by MDV DVL
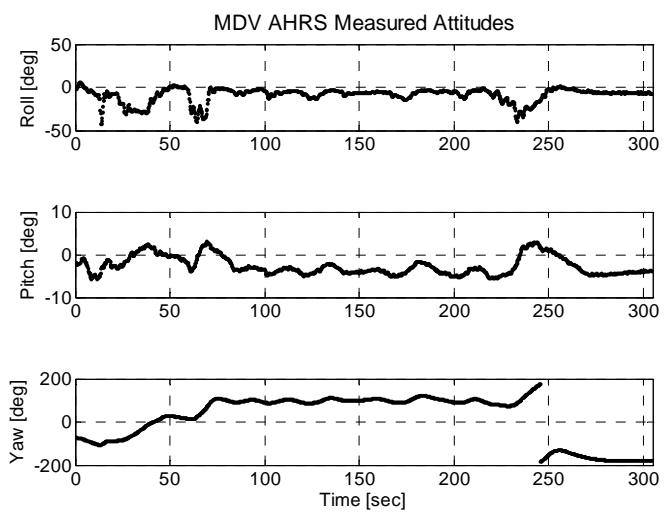

Fig. 7. Measured attitudes by MDV AHRS
Figs. 6 and 7 depict the measured sensor values of DVL and AHRS during the sea trial which was executed at Noryuk Island in the Jangheung province.

Figs. 8 and 9 show standalone dead reckoning results before and after heading alignment in the navigation frame. Because the data collected in the wide area and the track has a special figure, the heading alignment error can be identified. The standalone dead reckoning error sources are mainly due to a heading alignment mismatch between the body frame axis and the sensor frame axis.

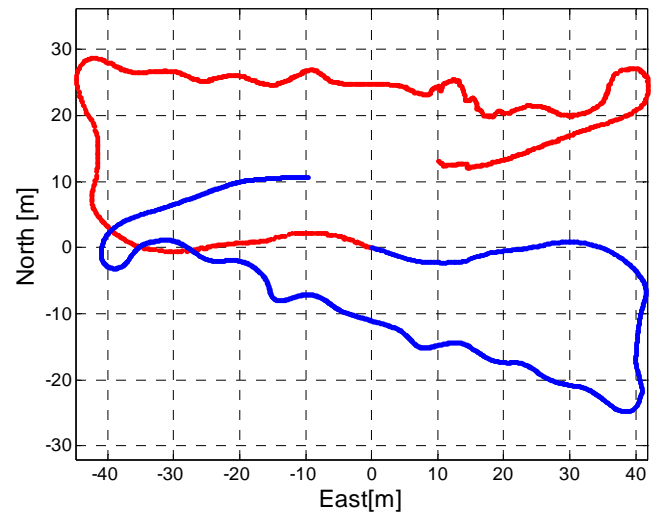

Fig. 8. Before sensor frame alignment against global coordinate

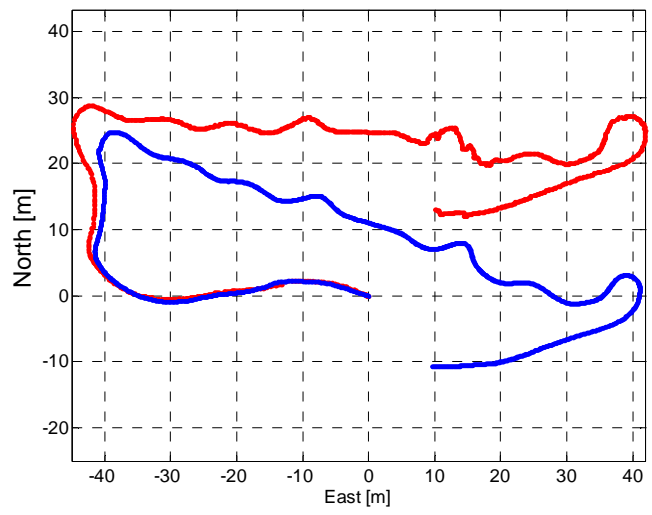

Fig. 9. After sensor frame alignment against global coordinate, DGPS (red), DR (blue) 


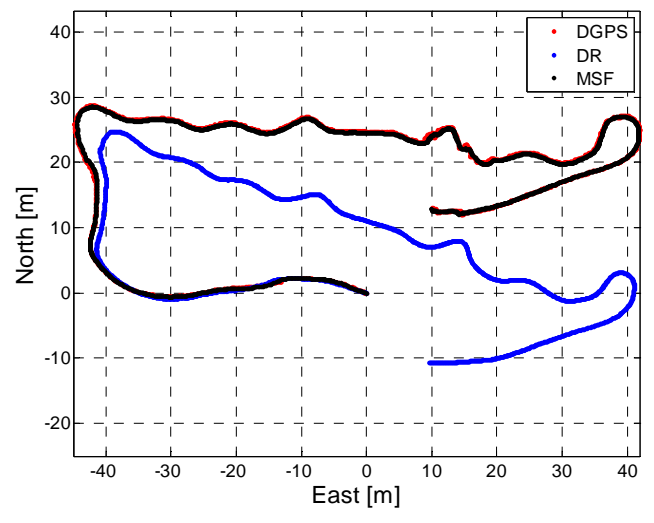

Fig. 10. Comparison of multi-sensor fusion results (black) with DGPS measurements (red) and pure dead-reckoning results (blue)

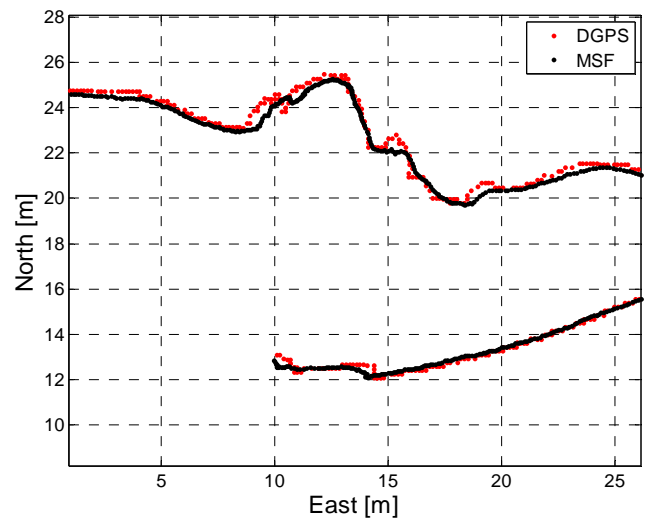

Fig. 11. Performance of multi-sensor fusion results (black) compared with DGPS measurements (red)

To prevent the accumulation of dead reckoning error, the outlier rejected USBL data can be used for accuracy enhancement.

Figs. 10 and 11 depict the MSF based navigation algorithm performance. Results show that the MSF algorithm generates precise estimates with good accuracies and $5 \mathrm{~Hz}$ update rate, which merges the merits of DR (DVL+AHRS) and DGPS (1Hz).

Fig. 11 compares the DGPS data and MSF algorithm results in the East and North coordinates. The MSF algorithm result shows a smoother and more precise trajectory than the stepwise DGPS track.

Fig. 12 depicts the errors of DR and MSF algorithms against the DGPS data. While the standalone DR scheme shows larger error and drift with time, the MSF algorithm results show a smaller and more bounded error.
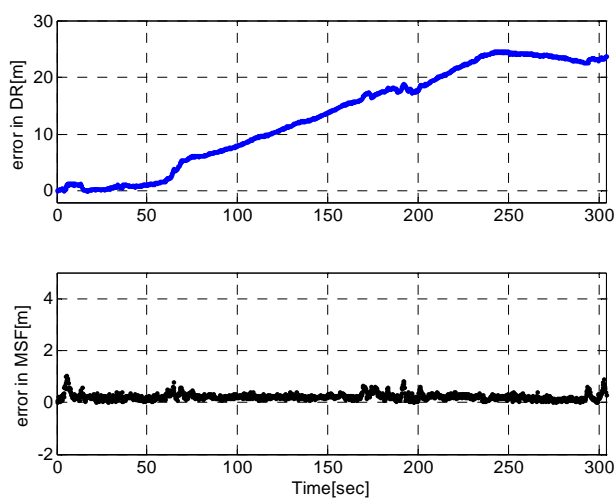

Fig. 12. Error analysis compared with DGPS measurements

\subsection{USBL, DVL, AHRS fusion algorithm}

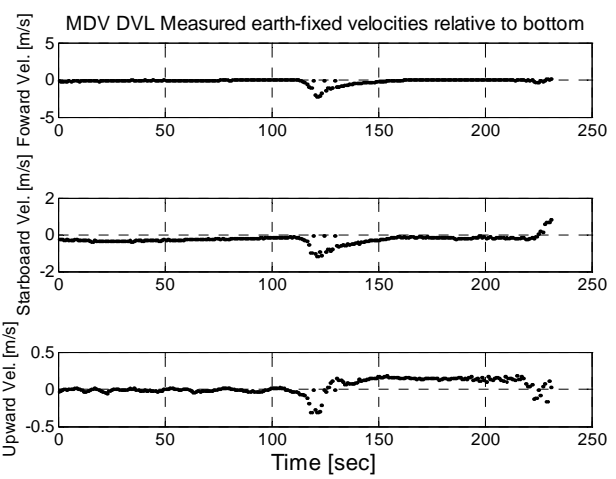

Fig. 13. Measured earth-fixed velocities by MDV DVL

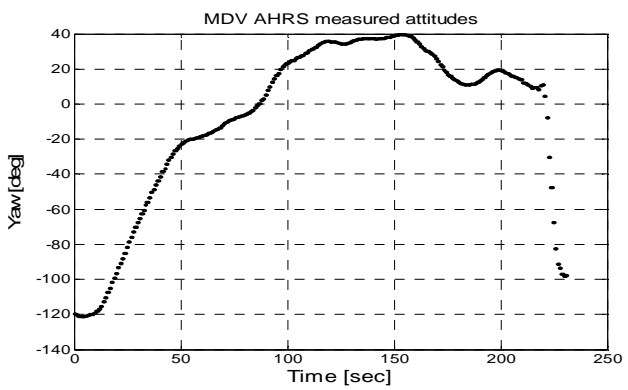

Fig. 14. Measured attitudes by MDV AHRS

Section 6.2 deals with the multi-sensor fusion algorithm for the underwater operation condition based on USBL, DVL and AHRS sensors.

Figs. 13 and 14 depict the measured sensor values of DVL and AHRS during the sea trial which was executed at the South-sea Bravo region. 
Figs. 15 and 16 show standalone dead reckoning results before and after heading alignment respectively.

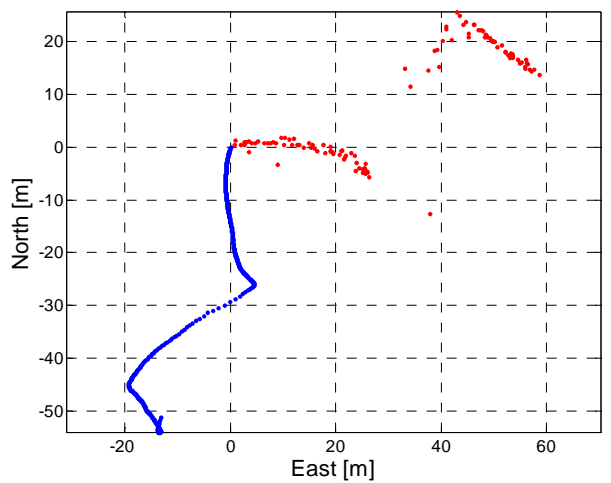

Fig. 15. Before sensor frame alignment against global coordinate

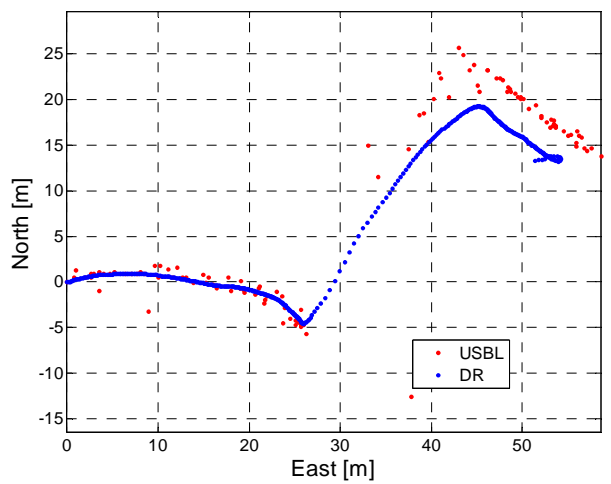

Fig. 16. After sensor frame alignment against global coordinate
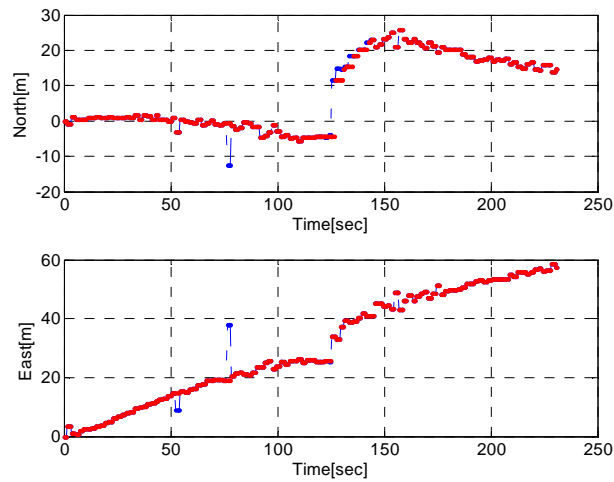

Fig. 17. Outlier rejection results (blue: raw data, red: outlier removed)

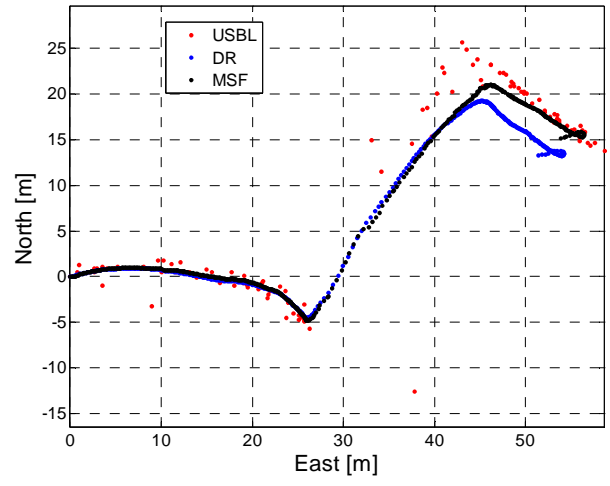

Fig. 18. Comparison of multi-sensor fusion results (black) with USBL measurements (red) and pure dead-reckoning results (blue) in navigation frame
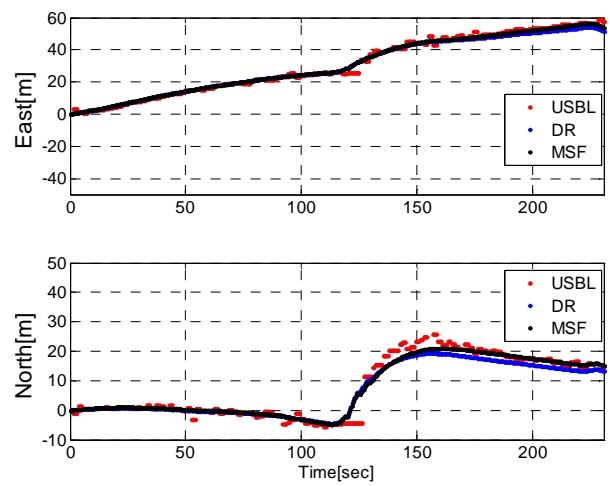

Fig. 19. Comparison of multi-sensor fusion results (black) with USBL measurements (red) and pure dead-reckoning results (blue) in time series
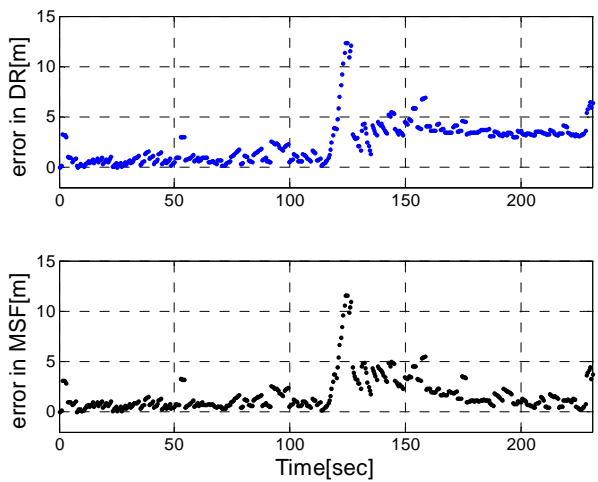

Fig. 20. Error analysis compared with outlier removed USBL measurements

Fig. 17 shows outlier rejection algorithm performance. Outliers outside the predefined gate as shown in Eq. (17) are removed effectively. 
Figs. 18 and 19 depict the MSF based navigation algorithm performance in a spatial frame and time series. Results show that the MSF algorithm estimates precise horizontal position updates with good accuracies and $5 \mathrm{~Hz}$ update rate while USBL measurements are obtained within 2 second period.

Fig. 20 depicts the errors of the DR and MSF algorithms against the outlier removed USBL data (not true value but considered as scattered trend). Especially, the errors at 125 seconds do not reflect the real situation. The USBL signal was lost temporarily because MDV moved too rapidly.

While the standalone DR scheme shows larger error and drift over time, the MSF algorithm results show a smaller and more bounded error.

The final error occurred because the USBL system's filter does not estimate rotational motion effectively. The authors assume that, even at that moment, the MSF algorithm can estimate actual MDV position robustly.

\section{Summary}

In this paper, the authors investigated the kinematic relations for the precise dead reckoning algorithm. In addition, the major error sources of a standalone DR algorithm are sought. From the kinematic relation and calculation results, the authors can conclude that the heading alignment angle should be identified and compensated with in-situ experimentally measured data.

The practical MSF algorithm for precise and accurate navigation in the horizontal navigation frame is designed and verified for the application of surface and underwater vehicle navigation. The developed algorithm is robust to outliers and is easy to implement as a real time navigation solution.

To demonstrate the performance of the designed MSF based navigation algorithm, online estimation with experiment data is carried out and verifying the MSF algorithm can be applicable for complicated MDV's mine hunting missions.

\section{Acknowledgements}

This research was supported by the ACTD projects, "Development of an auto cruising mine disposal vehicle", which financially supported by the DARPA of South Korea and "Development of core technologies for autonomous mapping of ocean bottom", which is supported by KORDI.

\section{References}

[1] L. Whitcomb, D. Yoerger and H. Singh, Advances in Doppler-Based Navigation of Underwater Robotic Vehicles, Proc. Of IEEE Int. Conf. on Robotics \& Automation, (1999) 399406.

[2] J.C. Kinsey and L.L. Whitcomb, In situ Alignment Calibration of Attitude and Doppler Sensors for Precision Underwater Vehicle Navigation: Theory and Experiment, IEEE Journal of Oceanic Engineering, 32(2) (2007) 286-299.

[3] J. Jouffroy and J. Opderbecke, Underwater vehicle trajectory estimation using contracting PDE-based observers, in American Control Conference, (2004) 4108-4113.

[4] S.Y. Cho and W.S. Choi, Robust Positioning Technique in Low-Cost DR/GPS for Land Navigation, IEEE Transactions on instrumentation and measurement, 55(4) (2006) 1132-1142.

[5] P. Lee, S. Kim, B. Jeon, H. Choi, and C. Lee, "Pseudo long base line navigation algorithm for underwater vehicles with inertial sensors and two acoustic range measurement," Ocean Eng., 34(3-4) (2007) 416-425.

[6] D. Fränken and A. Hüpper, Improved Fast Covariance Intersection for Distributed Data Fusion, Int. Conf. on Information Fusion, (2005) 154-160.

[7] S. Vike and J. Jouffroy, Diffusion-based outlier rejection for underwater navigation, Proc. of Oceans 2005, (2005) 1037-1042.

[8] W.S. Cleveland, Robust Locally Weighted Regression and Smoothing Scatter plots, Journal of the American Statistical Association, 74, (1979) 829-836.

[9] T. I. Fossen, Guidance and control of ocean vehicles, John Wiley \& Sons, 1994

[10]W. Niehsen, Information fusion based on fast covariance intersection filtering, In Proc. Int. Conf: Inf: Fusion (FUSION '02) (2002) 901905.. 